\title{
UTILIZAÇÃO DE ALGORITMOS GENÉTICOS EM GERAÇÕES DE DADOS DE TESTE DE SOFTWARE COM AGRUPAMENTO DE GENES E REDUÇÃO DE ESPAÇO DE BUSCA
}

\author{
Francisco Vitor Lopes da Frota, Walter Lucas Monteiro de Mendonça, \\ Daniel Alves da Silva, Márcio A. de Souza Fernandes, Nilton Freitas Barbosa e Edna Dias Canedo \\ Universidade de Brasília - Departamento de Engenharia Elétrica, Brasília - DF, Brasil - Zipcode 70910-900
}

\begin{abstract}
RESUMO
Uma das tarefas mais importantes para o desenvolvimento de software é a realização de testes que em boa parte das vezes é feita manualmente pelo testador ou desenvolvedor. O processo de teste pode ser facilitado por meio de automação sem a necessidade de intervenção humana e agilizando as tarefas de teste com a geração de dados automatizados. No entanto, a geração de dados de teste de forma automática pode dificultar esse processo em relação a criação e validação que podem necessitar de recursos externos e sobrecarregar o software. Este artigo apresenta uma solução simples utilizando algoritmo genético para a criação de dados com baixo número de gerações e com maior qualidade. A solução também mostrou eficiência em relação a não utilização de recursos externos e validação dos dados de teste gerados. A proposta supera algumas abordagens exploradas anteriormente.
\end{abstract}

\section{PALAVRAS-CHAVE}

Algoritmos Genéticos, Algoritmo de Diff Myers, Geração de Dados de Teste

\section{INTRODUÇÃO}

Testar softwares é de extrema importância para garantir sua qualidade durante o processo de desenvolvimento. As aplicações de testes podem ocasionar um aumento de custos e tarefas ao seu ciclo de vida útil. A maior parte dos esforços de desenvolvimento são alocados para a melhoria da qualidade, de acordo com (Pigoski, 1996). Comumente na indústria, a criação de dados deteste é realizada pelo testador através de um processo manual. Todavia, tal prática é altamente cara e trabalhosa e pode ser substituída pela automação de testes.

A automação de testes busca minimizar os custos de qualidade de software, mas existem fatores que aumentam a complexidade deste processo em comparação ao processo manual. Alguns fatores como: a necessidade de criar e manter scripts, gerar e validar a qualidade dos dados gerados. Dentre os fatores mencionados, destaca-se a criação de dados que pode ser realizada por ferramentas automáticas que utilizam técnicas de geração aleatórias ou semi-aleatórias, tais como as propostas por (Scalabrino et al., 2018) e (Frota et al., 2017). No entanto, essas alternativas podem não ser suficientes para a criação de dados com alta qualidade devido a sua natureza aleatória.

Este trabalho apresenta um gerador de dados que realiza a detecção automática de padrões extraídos dos sistemas por meio de um web scraper. O algoritmo realiza uma busca no sistema para gerar novos dados, que se assemelham aos armazenados, reduzindo o custo computacional e aumentando sua qualidade. Essa abordagem, diferente de outras encontradas na literatura, busca criar dados que se adaptem aos padrões do software.

O algoritmo se mostrou eficiente na criação de dados, necessitando de uma quantidade menor de gerações para alcançar resultados com alta qualidade sem a necessidade de utilizar recursos externos para validação e medição da qualidade. Foi observada uma taxa de erro abaixo de $10 \%$ para alguns dados, que foi reduzida aplicando técnicas especificas mencionadas neste trabalho. A proposta superou algumas abordagens exploradas anteriormente. 


\section{REFERENCIAL TEÓRICO}

Existem diversos tipos de abordagens para geração de dados de teste de forma automatizada. Conforme (Michael et al., 1997), alguns métodos para geração de dados são comumente utilizados para automatizar esse processo como a Geração Randômica, Geração Simbólica, Geração Dinâmica e Geração por Algoritmos Genéticos.

\subsection{Geração Randômica}

A geração de dados de forma randômica consiste em um método simples, que cria múltiplos dados aleatórios, até que um com qualidade alta seja alcançado segundo (Korel, 1990). O problema dessa abordagem é que, em programas complexos, um dado de teste adequado, pode ser difícil de ser encontrado, visto que a quantidade de dados adequados é inferior e a probabilidade de selecionar um dado com qualidade seja muito baixa de acordo com (Michael et al., 1997).

\subsection{Geração Simbólica}

Geração de dados de execução simbólica consiste em assinalar valores para variáveis, com o objetivo de satisfazer um critério de entrada adequado. Essa alternativa se mostra eficiente em sistemas que podem ser convertidos em abstrações matemáticas. Nesse ponto o algoritmo serve para resolver problemas com expressão algébrica segundo (Korel, 1996). Um dos usos comuns, para resolução desses problemas, é a criação de um laço de repetição com diferentes respostas variando os parâmetros. O problema ocorre quando se tenta obter todas as respostas possíveis, e o loop pode não entrar na solução correta, de forma que o problema tende ao infinito em seu tempo de processamento conforme (Michael et al., 1997).

\subsection{Geração Dinâmica}

A geração de dados dinâmica, de acordo com (Korel, 1996), é baseado na ideia de que as funcionalidades de um sistema podem ser substituídas por funções programáticas. O objetivo é executar tal função com diversos parâmetros, até que um certo ponto do código seja atingido e, assim, modificar um ou mais variáveis da função até que ela atinja o seu final. A função de avalição é bastante complexa, mas pode ser avaliada por qualquer entrada. O problema de gerar dados de teste com essa abordagem, é o grau de dificuldade para reduzir o problema a uma função de minimização conforme dito por (Michael et al., 1997).

\subsection{Geração por Algoritmos Genéticos}

Algoritmos genéticos (AG) são métodos de otimização inspirados nos modelos de seleção natural e genética e podem ser aplicados a uma grande quantidade de problemas, inclusive problemas práticos do dia a dia de acordo com (Coley, 1999). Dentro de um AG o termo cromossomo é usado para indicar uma possível solução, representado geralmente como uma sequência binária.

Segundo (Koza, 1997), um cromossomo é criado a partir de múltiplos genes, se o cromossomo for uma solução em um formato binário, o gene vai ser um"0"ou"1". As soluções são obtidas de uma lista possivelmente infinita de possíveis soluções, conhecida como espaço de busca, que tem como objetivo alcançar a resolução de um problema.

O termo fitness, indica a qualidade potencial do cromossomo para resolver um problema preestabelecido. Essa qualidade é representada de uma forma numérica, por vezes binária, sendo resultado de uma função de fitness de acordo com (Booker et al., 1989; Deb et al., 2002). O fitness máximo a ser alcançado é denominado máximo global, enquanto os picos menores são denominados máximos locais. Para algumas aplicações, por vezes, alcançar o máximo global não é necessário para resolver um problema, desde que a solução tenha uma qualidade mínima para aquele problema em específico. As operações básicas de um algoritmo genético incluem seleção, crossover e mutação conforme (Deb et al., 2002). 
Seleção é o momento em que os cromossomos são selecionados dentro de uma população, simulando o processo de seleção natural. A primeira população é geralmente composta por cromossomos aleatórios conforme (Bodenhofer, 2003). Os indivíduos são classificados com base em seu fitness, e aqueles que possuem pontuação menor têm maior probabilidade de serem descartados.

Segundo (Koza, 1997), crossover é baseado na reprodução animal, a genética dos dois elementos são divididos aleatoriamente e misturados para gerar um novo indivíduo. Assim elementos que tenham alta qualidade podem gerar progênitos com uma maior qualidade, ou mesmo melhor que seus progenitores. De acordo com (Holland et al., 1992), mutação é o processo que garante a variação e inovação do AG, quando múltiplos genes são alterados para manter a diversidade genética.

\section{TRABALHOS RELACIONADOS}

Alguns trabalhos encontrados na literatura, foram utilizados para comparação com a solução aqui proposta. A solução proposta por (Chang et al., 1996) utilizou em um primeiro momento a geração randômica que obteve a pior performance (alto tempo de execução) alcançando uma cobertura de 93,4\% no melhor caso. Todavia, se a complexidade do programa for aumentada, a cobertura decai para $45,5 \%$.

A pesquisa realizada por (Korel, 1996) compara algumas abordagens e relata que, utilizando a geração randômica foi alcançado no máximo $79 \%$ de cobertura. Com a geração simbólica alcançou $90 \%$ e por fim, utilizando a abordagem dinâmica, foi possível alcançar aproximadamente $99 \%$ de cobertura para três programas distintos.

Diferentemente da solução proposta por (Korel, 1996), a geração de dados utilizando AG de (Michael et al., 1997) visa minimizar um teste para uma função numérica. Para realizar a avaliação, é necessário fazer uma abstração do problema para uma função, que exige conhecimento de um especialista, dificultando a execução deste processo. Para os resultados deste trabalho, utilizando a abordagem randômica foi alcançado $77 \%$ de cobertura.

Diante dos trabalhos mencionados, a pesquisa atual propõe uma abordagem distinta, simplificada e de fácil aplicação. A proposta e os passos seguidos serão apresentados na seção a seguir.

\section{PROPOSTA DE SOLUÇÃO}

A criação de valores representa uma parte significativa do planejamento de testes de tal forma que motive os esforços feitos para a automação dessa tarefa. Algoritmos Genéticos já foram utilizados em diversos estudos para a criação de dados, como mostrado na seção anterior. No entanto, a abordagem proposta neste artigo abstém-se da execução de testes diretamente no sistema, como uma forma de validação, dando ênfase em sua comparação entre os dados.

Entre algumas desvantagens ao se utilizar o próprio sistema para validar os dados, estão:

- Depende totalmente da disponibilidade do sistema para que os dados sejam inseridos, enviando requisições que podem demandar muito tempo de processamento;

- O sistema pode requisitar recursos externos como banco de dados, web servisse e outros módulos, necessitando de conexão via internet, que podem demandar muito tempo para a execução dos testes e validação dos dados.

A solução proposta usa valores mapeados, comparando um(K)valor qualquer com os valores já inseridos no sistema ao invés de utilizar o sistema em si. Para a geração dos valores, a abordagem apresentada é semi-aleatória, baseada em padrões existentes aumentando a possibilidade de um gene novo ser usado na criação de um novo indivíduo. A Figura 1 exibe os passos seguidos para chegar ao melhor resultado de acordo com essa solução. 


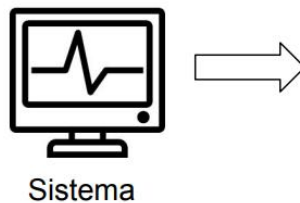

Sistema

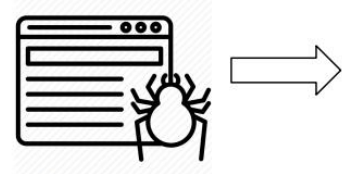

Scraper

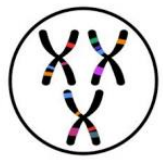

Geração

de dados

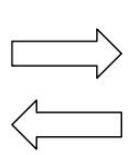



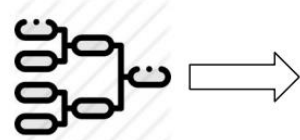

Validação

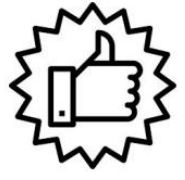

Melhor resultado

Figura 1. Fluxo de execução da abordagem

No sistema, são armazenados os valores já cadastrados com sucesso, que serviram de base para a criação de novos dados pelo algoritmo genético. Esses valores são extraídos por meio de um scraper, e o reconhecimento dos objetos de teste a serem extraídos é feito por uma redeneural1.

A partir dos dados extraídos, é criada uma base de dados contendo os caracteres e a proporção de uso de cada um. A partir dessa base de caracteres, novos indivíduos são gerados pelo algoritmo genético, passando pelos processos de criação de novas gerações, crossover e validação.

A validação é realizada por meio da comparação do dado atual gerado com os outros dados anteriormente armazenados na base. Essa comparação gera uma pontuação de fitness que diz o quão adequada um dado de teste é para um campo de entrada.

O algoritmo de cálculo de fitness, se baseia no cálculo da diferença do indivíduos atual com todos os outros da base. O algoritmo utilizado para realizar a validação é o difference algorithm criado por (Myers, 1986). O Algoritmo retorna o valor da diferença entre dois conjuntos de caracteres.

A diferença entre os indivíduos da população é convertida para semelhança, subtraindo a pontuação da diferença da quantidade total de caracteres. A semelhança é calculada para todos os indivíduos da base junto com o atual, logo em seguida, é computada a média da semelhança entre todos os indivíduos.

Por meio dos passos mencionados nesta seção, foi conduzido um experimento para validar os resultados da solução proposta. Tais resultado serão apresentados na seção a seguir.

\section{RESULTADOS}

Através dos resultados, foi possível validar a qualidade de dados de teste gerados usando algoritmos genéticos. Os tipos de dados utilizados foram E-mail, nome e data, servindo de ponto departida para a geração de variáveis mais complexas. A Tabela 1 mostra os resultados do fitness calculado para alguns dados de data. Podemos observar que os dois primeiros valores são dados inválidos, recebendo um valor de fitness baixo em relação ao terceiro valor, cujo valor é alto identificando um dado adequado.

Tabela 1. Descrição dos dados usados para validação do algoritmo

\begin{tabular}{cc}
\hline Dado de teste & Fitness \\
\hline $39-20-3115$ & 64 \\
$38-19-2016$ & 48 \\
$08-04-2016$ & 85 \\
\hline
\end{tabular}

O algoritmo genético usado para a criação dos valores usa uma abordagem de torneio e mutação. A taxa de mutação é o operador usado para diversificar uma geração de outra. Para este experimento, o de mutação equivale a0.015e limitando uma quantidade máxima de 60 gerações. Um valor baixo é usado para que os ganhos de fitness obtidos na geração não sejam perdidos, por outro lado, usar um valor mais baixo que esse faria com que o algoritmo ficasse preso em indivíduos de qualidade menor em maior frequência de acordo com (Srinivas and Patnaik, 1994). Os parâmetros foram selecionados ao executar diversas variações entre o conjunto, escolhendo as melhores entre eles. Os resultados de qualidade dos parâmetros podem ser observados na Figura 2. 


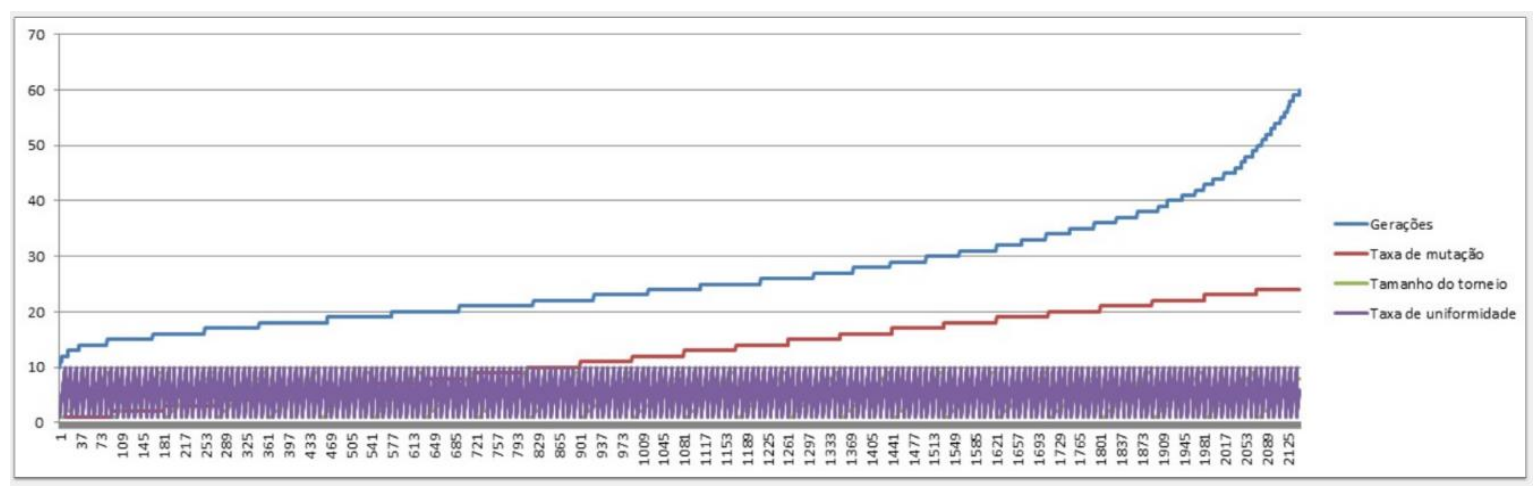

Figura 2. Correlação entre a variação dos parâmetros do algoritmo genético e a qualidade final

A partir dos resultados apresentados na Figura 2, foi possível identificar o conjunto de variáveis com maior performance. A taxa de uniformidade apresentou uma variação que não ocasionou impacto na qualidade final do fitness, variando apenas entre 1 e 10 ao decorrer da execução.

Em relação à quantidade de gerações e ao nível de fitness dos dados de teste, foi possível aferir que a maior parte do aumento da qualidade ocorre nas primeiras gerações, sendo que as gerações subsequentes servem apenas para aprimorar ainda mais a qualidade, não sendo necessárias na maioria dos casos.

Foram identificados alguns padrões para os dados utilizados no experimento. A data, por padrão, usa números de 1 a 31 para representar os dias do mês, de 1 a 12, para representar os meses do ano e quatro dígitos positivos representando os anos. Para e-mail, são utilizados uma sequência de caracteres, seguidas por '@' e por um domínio de e-mail válido. O nome serve para validar uma cadeia de caracteres intercaladas por espaço. Esses dados foram escolhidos por existirem em muitos dos formulários de cadastro de usuário e também por terem meios distintos de validação como, por exemplo, sequências numéricas com padrões simples, padrões alfanuméricos que contem partes fixas e variáveis.

A implementação do algoritmo foi realizada utilizando a linguagem Java, sendo executada em um computador com processador I5 e8 gigas de memória RAM. O algoritmo para cada valor foi executado com um número máximo de 60 gerações, ou até atingir um padrão máximo de qualidade. Esse padrão de qualidade é atingido quando há 5 gerações sem melhora ou o indivíduo com mais fitness dentre 60 gerações. A Tabela 2 detalha os resultados da execução do algoritmo, sendo a quantidade de erros, o número de gerações até alcançar o melhor resultado, e por fim, o tempo de execução em Milissegundos.

Tabela 2. Resultado de execução dos testes do algoritmo

\begin{tabular}{cccc}
\hline Dado & Erro & Gerações & Tempo Execução $(\mathbf{m s})$ \\
\hline E-mail & 23 & 13 & 3188 \\
Nome & 0 & 24 & 2293 \\
Data & 7 & 7 & 650 \\
\hline
\end{tabular}

A massa de dados usada para gerar os E-mails foi dividida em três domínios, $50 \%$ com o domínio do 'Gmail' 25\% com domínio de 'Yahoo' e 25\% com o domínio de 'Hotmail'. Antes de executar o algoritmo genético, foram selecionados da base, dados com as características predominantes, ou seja, no caso específico para e-mails, com a mesma quantidade de caracteres que apareçam mais vezes são selecionados para serem usados pelo algoritmo genético. Com a execução do algoritmo, foi observado que apenas $23 \%$ de $100 \%$ dos dados gerados, foram descartados por terem baixa qualidade.

Para a geração de dados de data, foram obtidos 1700 valores no formato DD/MM/AAAA, entre o período de 01/01/2015 a 01/01/2016. Para esses resultados, uma taxa de erro de 7\%. Existem duas causas distintas para explicar os erros. A primeira consiste em dados com números acima de 31 para dias. A segunda, consistiu em números que não eram válidos para os meses. Dentre os 7 valores errados, 5 foram causados por números inválidos no campo mês. 
Esse problema foi resolvido estipulando um fitness mínimo de 80 pontos para que o dado seja utilizado em outra geração. Caso o dado não consiga evoluir em um número preestabelecido de gerações e não contenha uma quantidade mínima de qualidade, o algoritmo será executado novamente até alcançar uma qualidade aceitável. Por fim, os dados do tipo nome contêm menos restrições que os demais apresentados, pois são considerados válidos quando há uma sequência de caracteres intercalada por espaços. Para os nomes, não foram apresentados erros, pois o problema é bastante simples.

A alternativa padrão de AG é utilizar uma quantidade maior de caracteres que contemplem os diversos problemas, incluindo: letras, números e símbolos, que resultam em 256 caracteres. De acordo com os resultados apresentados, é possível inferir que a alternativa apresentada aumenta a velocidade de execução, como no caso da geração de datas, o tempo de execução foi reduzido em $95.4 \%$.

\subsection{Comparação entre as Abordagens com os Resultados Atuais}

Ao observar a Tabela 3, foi possível aferir que a solução proposta, ao utilizar a combinação de AG e Myers' Diff Algorithm para o cálculo de fitness, mostrou-se mais performática e gerou dados com maior qualidade, superando a maioria das abordagens exploradas em trabalhos anteriores. O resultado foi muito próximo do obtido por Chang et al. (1996).

Tabela 3. Comparação entre as soluções disponíveis na literatura e a proposta neste trabalho

\begin{tabular}{ccc}
\hline Autor & Abordagem & Porcentagem acerto \\
\hline [(Chang et al., 1996)] & Randômica & $41 \%$ \\
[(Michael et al., & Genética & $60 \%$ \\
1997)] & Randômica & $77 \%$ \\
[(Michael et al., & Randômica \\
1997)] & (Baixa performance) & $93,4 \%$ \\
[(Chang et al., 1996)] & Randômica & $67 \%$ \\
{$[($ Korel, 1996)] } & Simbólica & $63 \%$ \\
[(Korel, 1996) & Genética + Myers' Diff Algorithm & $93 \%$ \\
\hline Este trabalho & &
\end{tabular}

Todavia, o tempo para execução e geração dos dados foi altamente elevado, diferentemente da solução apresentada neste artigo. O autor apenas relata que o tempo para a geração foi consideravelmente alto, mas assim como as outras propostas comparadas, não foram relatados tempo de execução, impossibilitando a comparação em relação ao tempo de processamento.

Essa abordagem, também se mostrou eficiente em relação a geração de dados com maior qualidade em poucas gerações, mostrando que a combinação com o algoritmo resultou em benefícios para a geração de dados de teste.

\section{DISCUSSÕES}

Ao aplicar a técnica de algoritmos genéticos para a geração de dados de teste, foi possível observar que substituição de processos manuais por AG pode minimizar esforços e produzir dados com alta qualidade, sem a necessidade de interação humana, e também podem ser validados sem o uso de recursos externos que podem prejudicar o funcionamento normal do software testado.

Esse método se mostrou eficiente em simular a criação de dados com complexidade moderada em valores alfanuméricos, sem dependências entre seus caracteres. Por meio dos experimentos realizados, alguns resultados mostram que é possível melhorar o tempo em relação ao processo manual. Foi possível aferir que a utilização de algoritmos como o Diff Myers melhora a qualidade dos dados e que a proposta supera algumas abordagens realizadas anteriormente. 


\section{TRABALHOS FUTUROS}

Como trabalho futuro para o aprimoramento da geração de dados de teste, deseja-se identificar padrões mais complexos em que os diferentes fragmentos de dados sejam dependentes um do outro, tais como: dados que usam fórmulas matemáticas para serem criados como CPFs e números de cartão de crédito. Outra possibilidade seria realizar a comparação com mais técnicas e algoritmos encontrados na literatura, com o intuito de validar ainda mais a eficiência da solução proposta atualmente.

\section{AGRADECIMENTO}

Os autores agradecem o apoio das Agências brasileiras de pesquisa, desenvolvimento e inovação CNPq (Projeto INCT SegCiber 465741/2014-2), CAPES (Projetos FORTE 23038.007604/2014-69 e PROBRAL 88887.144009/2017-00) e FAPDF (Projetos UIoT 0193.001366/2016 e SSDDC 0193. 001365/2016), bem como o suporte do Laboratório LATITUDE/UnB (Projeto SDN 23106. 099441/2016-43), e as cooperações com o Ministério da Economia (TEDs DIPLA 005/2016 e ENAP 083/2016) e o Gabinete de Segurança Institucional da Presidência da República (TED 002/2017).

\section{REFERÊNCIAS}

Bodenhofer, U. (2003). Genetic algorithms: theory and applications.

Booker, L. B., Goldberg, D. E., and Holland, J. H. (1989). Classifier systems and genetic algorithms. Artificial intelligence, 40(1-3):235-282.

Chang, K. H., CROSS II, J. H., Carlisle, W. H., and Liao, S.-S. (1996). A performance evaluation of heuristics-based test case generation methods for software branch coverage. International Journal of Software Engineering and Knowledge Engineering, 6(04):585-608.

Coley, D. A. (1999).An introduction to genetic algorithms for scientists and engineers. World Scientific Publishing Company.

Deb, K., Pratap, A., Agarwal, S., and Meyarivan, T. (2002). A fast and elitist multiobjective genetical gorithm: Nsga-ii. IEEE transactions on evolutionary computation, 6(2):182-197.

Frota, F. V. L. d., Silva, D. A., Oliveira, R. A., Canedo, E. D., De Sousa, R. T. (2017). Uso de aprendizado de máquina para a automação de testes de sistemasweb.

Hansen, L. K. and Salamon, P. (1990). Neural network ensembles. IEEE Transactions on Pattern Analysis \& Machine Intelligence, (10):993-1001.

Holland, J. H. et al. (1992). Adaptation in natural and artificial systems: an introductory analysis with applications to biology, control, and artificial intelligence. MIT press.

Korel, B. (1990). Automated software test data generation. IEEE Transactions on software engineering, 16(8):870-879.

Korel, B. (1996). Automated test data generation for programs with procedures. In ACM SIG-SOFT Software Engineering Notes, volume 21, pages 209-215. ACM.

Koza, J. R. (1997). Genetic programming.

Michael, C. C., McGraw, G. E., Schatz, M. A., and Walton, C. C. (1997). Genetic algorithms for dynamic test data generation. In Proceedings 12th IEEE International Conference Automated Software Engineering, pages 307-308. IEEE.

Myers, E. W. (1986). Ano (nd) difference algorithm and its variations. Algorithmica, 1(1-4):251-266.

Pigoski, T. M. (1996). Practical software maintenance: best practices for managing your software investment. Wiley Publishing.

Scalabrino, S., Grano, G., Di Nucci, D., Guerra, M., De Lucia, A., Gall, H. C., and Oliveto, R. (2018). Ocelot: a search-based test-data generation tool for c. InASE, pages 868-871.

Srinivas, M. and Patnaik, L. M. (1994). Adaptive probabilities of crossover and mutation ingenetic algorithms. IEEE Transactions on Systems, Man, and Cybernetics, 24(4):656-667. 